\title{
Potential overfeeding among formula fed Special Supplemental Nutrition Program for Women, Infants and Children participants and associated factors
}

\author{
Christopher E. Anderson $^{1}{ }^{\infty}$ | Catherine E. Martinez ${ }^{1}$ | Alison K. Ventura ${ }^{2}$ | \\ Shannon E. Whaley ${ }^{1}$
}

${ }^{1}$ Department of Research and Evaluation, Public Health Foundation Enterprises (PHFE) WIC, Irwindale, California, USA

${ }^{2}$ Department of Kinesiology and Public Health, College of Science and Mathematics, California Polytechnic State University, San Luis Obispo, California, USA

Correspondence

Christopher E. Anderson, PhD, Research Analyst, Public Health Foundation Enterprises (PHFE) WIC, 12781 Schabarum Ave, Irwindale, CA 91706.

Email: christophera@phfewic.org

\section{Summary}

Background: The Special Supplemental Nutrition Program for Women, Infants and Children (WIC) provides enough formula to meet the known nutritional needs of infants up to 6 months of age whose mothers report not breastfeeding, but many mothers report WIC providing insufficient formula, indicating potential overfeeding. Objective: To estimate the prevalence of potential overfeeding among formula-feeding WIC participants and identify associated factors.

Methods: Potential overfeeding was identified among participants of the longitudinal Infant and Toddler Feeding Practices Study-2 (ITFPS-2) receiving the fully formulafeeding WIC infant package at 1 month of age $(n=1235$, weighted $n=197079$ ). Associations of potential overfeeding with caloric intake, weight and participant characteristics were assessed.

Results: Potential overfeeding was identified among $37.41 \%(95 \% \mathrm{Cl}=33.57-$ $41.25 \%)$ of fully formula-feeding infants. Potentially overfed infants were $0.18 \mathrm{~kg}$ heavier $(P$-value $=.01)$, consumed 26 more calories daily $(P$-value $=.004)$ and were more likely Non-Hispanic White or English-speaking Hispanic $(P$-value $=.007)$ and highly active at 5 months of age $(P$-value $=.01)$. Mothers of potentially overfed infants were less likely to agree that breastfeeding is easier than bottle feeding, only mothers can feed breastfed infants, turning away from the bottle indicates satiation, and crying always indicates hunger ( $P$-values $.04, .002, .04$ and .04 respectively), and more likely to report WIC provides insufficient formula early (1-5 months, $P$-value $<.0001)$ and late (6-13 months, $P$-value $=.007)$ in infancy.

Conclusions: Potential overfeeding occurs in $37 \%$ of fully formula-feeding infant WIC-participants $<6$ months old. Mothers of these infants may benefit from additional education about the formula needs of their infants and how to recognize infant satiation cues.

KEYWORDS

formula, overfeeding, WIC 


\section{1 | INTRODUCTION}

Rapid weight gain during the first 2 years after birth is robustly associated with heavier weight status later in childhood. ${ }^{1}$ Childhood obesity is associated with increased risk of adult obesity and with a number of chronic health conditions..$^{2-4}$ Obesity is highly prevalent among United States children, ${ }^{5}$ and more prevalent among children of low-income households. ${ }^{6}$ There is extensive research on nutritional causes of early childhood overweight and obesity, ${ }^{7}$ with lower obesity/overweight risk reported among children fed breastmilk instead of formula, ${ }^{8}$ who are breastfed instead of bottle fed, ${ }^{9}$ and for whom complementary foods and beverages (CFB) were introduced according to recommendation as opposed to earlier than recommended. ${ }^{10}$

The Special Supplemental Nutrition Program for Women, Infants and Children (WIC) is a federal nutrition assistance program that provides nutrition education, healthy foods, breastfeeding support and service referrals to pregnant or postpartum women and children under the age of 5 years in households with incomes below $185 \%$ of the federal poverty level (FPL). ${ }^{11}$ WIC promotes breastfeeding among enrolled postpartum women, however, a majority of participating women with infants 2 months of age or older receive the fully formula-feeding package. ${ }^{12}$ Among WIC-participating children, the risk of obesity is proportional to the number of months they were enrolled in an infant package that provides formula, with children fully formula fed from birth to 12 months of age having the highest risk of obesity at 4 years of age. ${ }^{13}$ The fully formula-feeding infant package provided by WIC is designed to provide enough formula to meet all known nutritional needs for the infants receiving it between 0 and 5.9 months of age. ${ }^{14}$ However, many WIC-participating mothers report needing more formula than is provided, ${ }^{15}$ which may suggest that some fully formula-fed WIC-participating infants are being fed more than is recommended or the formula provided is being wasted.

The Infant and Toddler Feeding Practices Study-2 (ITFPS-2) is a national and longitudinal study of WIC participating mothers and children up to 6 years of age designed to assess feeding practices and the health behaviors of children enrolled in WIC. ${ }^{16}$ The present study was designed to assess the prevalence of potential overfeeding among fully formula-fed WIC-participating infants in ITFPS-2, to assess longitudinal differences in caloric intake and infant weight between infants experiencing and not-experiencing potential overfeeding up to 36 months of age, and to identify maternal or child traits and feeding behaviors associated with potential overfeeding.

\section{2 | METHODS}

\section{1 | Participants}

Study participants were drawn from the longitudinal ITFPS-2, which was designed to be representative of the study-eligible national population of WIC-participants during the study recruitment period. ${ }^{16}$ The study strategy for sampling pregnant or newly postpartum ( $<2.5$ months) women has been described in detail in prior publications. ${ }^{17}$ Mothers were eligible for enrollment in ITFPS-2 if they were enrolling in WIC for the first time (for the specified pregnancy or infant), were 16 years of age or older, were not currently in foster care, were not a foster parent to the enrolled infant, and were able to complete English or Spanish telephone interviews. Infants enrolled in ITFPS-2 ( $N=3777)$ were included in this study if they were part of the core sample, were receiving the fully formula-feeding infant package at 1 month of age and were reported by their mother to be receiving only formula at 1,3 or 5 months of age (unweighted $n=1235$, weighted $n=197$ 079). Replicate sample weights were used to adjust for differential sampling and response rates to ensure that the estimates derived from this analysis were unbiased estimates for the entire source population of WIC-participants from the $80 \mathrm{WIC}$ sites included in the sampling frame. ${ }^{18,19}$ Longitudinal data were available for the included infants up to 36 months of age.

\section{2 | Potential overfeeding}

Infants receiving the fully formula-feeding infant package are provided with 403 and $442 \mathrm{fl} \mathrm{oz}$ of formula by WIC monthly from 0 to 3.9 and 4.0 to 5.9 months, respectively, ${ }^{20}$ which is enough to meet all of their known nutritional needs before the introduction of CFB. ${ }^{14}$ These infants enrolled in the fully formula-feeding package were identified as experiencing potential overfeeding (ie, consuming formula in excess of what WIC provides) if their mother reported that they were getting formula for the infant from WIC and another source in any of the surveys conducted at 1 (15.69\% of respondents), 3 (23.89\% of respondents) or 5 (27.34\% of respondents) months of age. WIC-participating infants with a medical condition that requires special nutritional guidance receive therapeutic formula packages and were not included in this study.

\section{3 | Outcomes}

Additional outcomes of interest in this study, besides potential overfeeding, were daily calories consumed and infant weight. Daily calories were calculated from 24-hour dietary recalls collected as part of each survey of the study participants between 1 and 36 months of age. Dietary recalls were administered over the phone, using the USDA Automated Multiple Pass Method. ${ }^{21}$ Details of the dietary intake assessment have been published previously. ${ }^{22}$ Infant weight was measured in grams, and was available for $84 \%$ of study participants at two or more times between birth and 36 months of age. Infant weight was available from WIC administrative data, measured by trained WIC staff, for infants who continued to participate in WIC. For infants who were no longer enrolled in WIC, attempts were made to collect weight from the infant's healthcare provider, during a home health agency visit arranged as part of the study, or by asking the 
mother to return to a WIC clinic to have her infant's weight measured.

\subsection{Covariates}

Variables that were available for inclusion in this study included child, maternal, and household characteristics. Children were characterized by demographic characteristics including sex, race/ethnicity (non-Hispanic Black, non-Hispanic White, non-Hispanic Other, Spanish speaking Hispanic and English speaking Hispanic), in addition to dichotomous variables for birth factors including low birthweight, delivery $\geq 3$ weeks preterm, Cesarean section, and any delivery complications. English and Spanish speaking Hispanics were separated in the race/ethnicity categorization due to prior associations between language preference and infant feeding choices ${ }^{13}$ and WIC enrollment and retention. ${ }^{23}$ Frequency of infant activity was assessed by maternal report at 5 (tummy time, rolling around, playing with a ball) and 13 (play wrestling, chasing, tumbling, playing with a ball) months of age (everyday, several times per week, once per week, or not at all). Activity scores were calculated at each point by adding 3 points for each "everyday" activity, 2 points for each "several times per week" activity, 1 point for each "once a week" activity and 0 points for "not at all" activities. A variable for high activity was created at each age (scores $\geq 5$ at 5 months, scores $\geq 7$ at 13 months). Mothers were characterized by marital status (yes or no), co-habitation status (yes or no), overweight/ obesity status at screening (yes or no), prior parity (yes or no), breastfeeding history (any or none), timing of enrollment in WIC (first, second or third trimester of pregnancy or postnatally), age at delivery ( $<20$ or $\geq 20$ years), education (<high school, high school or $>$ high school) and receipt of other government benefits (any or none). Households were characterized with income $(<75 \%$ FPL, $75-130 \%$ FPL or $>130 \%$ FPL) and food security status (very low, low or high/marginal).

Non-recommended bottle feeding practices were characterized by dichotomizing (ever or never) responses to questions about the frequency of mixing formula with extra water, mixing formula with less water, adding bottle sweetener, using a bottle with an extra-large nipple hole, propping up the infant's bottle, encouraging the infant to finish the contents of the bottle, and feeding on a schedule. Total and formula-specific daily feeding frequency were characterized continuously by maternal report in 24 -hour dietary recalls at 1,3 and
5 months of age. Mothers provided information about individual contacts that provided input about infant feeding decisions including her boyfriend/husband, her mother, other relatives, friends, WIC staff or a physician. Responses were coded as yes or no, and the number of categories of contacts was calculated by adding 1-point for each affirmative response. Breastfeeding intent was assessed with the Infant Feeding Intentions scale. ${ }^{24}$ Mothers responded to questions about the strength of agreement with a series of questions about infant feeding and cues on a Likert scale (strong disagreement, disagreement, neutrality, agreement and strong agreement), which were then dichotomized (strong agreement and agreement vs neutral, disagreement and strong disagreement). Maternal perception of the amount of formula provided by WIC was assessed in each survey (when infants were $1,3,5,7,9,11$ or 13 months of age). A dichotomous variable indicating that WIC provides insufficient formula was created for early infancy (1, 3 or 5 months) and late infancy (7, 9, 11 and 13 months) if a mother reported that the amount of formula provided by WIC was not enough for any survey in either age period.

\section{5 | Analysis}

All statistical analyses were weighted with ITFPS-2 sample weights for the core-sample at the 1-month interview (when the fully formula feeding WIC infant package was assessed) to accommodate unequal sampling rates and nonresponse. Differences in the number of daily kilocalories consumed and infant weight between infants experiencing and not experiencing potential overfeeding were assessed longitudinally with mixed effects models that were adjusted for infant characteristics identified a priori as potential confounders (sex, age) $)^{25,26}$ or that differed between infants experiencing and not experiencing potential overfeeding (preterm birth, high activity at 5 months, low birthweight, race/ethnicity), ${ }^{27}$ incorporating sampling weights, random intercepts and a random effect for age. An interaction of age with the potential overfeeding variable was included to assess differences in the rate of change for daily caloric intake and weight by potential overfeeding status, but these terms were removed when they were found to be non-significant. Results of a sensitivity analysis excluding preterm infants did not differ from the main results, and are therefore not presented. Infants identified as experiencing and not experiencing potential overfeeding were characterized with frequencies or means

TAB LE 1 Daily energy consumption and weight for WIC-participating formula feeding infants experiencing potential overfeeding and nonoverfeeding in ITFPS-2 ( $\mathrm{N}=197$ 079)

\begin{tabular}{llll} 
Variable & $\begin{array}{l}\text { Potential Overfeeding } \\
\boldsymbol{\beta} \text { (SE) }\end{array}$ & $\begin{array}{l}\text { Non-overfeeding } \\
\boldsymbol{\beta} \text { (SE) }\end{array}$ & $\boldsymbol{P}$-value \\
\hline Daily calories (kilocalories) $^{\mathrm{a}}$ & $26.10(8.94)$ & Ref. $^{\mathrm{s}}$ & .004 \\
Weight (kilograms) $^{\mathrm{b}}$ & $0.18(0.07)$ & Ref. & .01 \\
\hline
\end{tabular}

Note: Bold values indicate statistical significance $(P<0.05)$.

aLongitudinal mixed effects model was adjusted for low birthweight, preterm delivery, high activity at 5 months of age, race/ethnicity, child sex and age. Model also included random intercept and random effect for age. Group difference in reported daily caloric intake is estimated at 18 months.

${ }^{b}$ Longitudinal mixed effects model was adjusted for low birthweight, preterm delivery, high activity at 5 months of age, race/ethnicity, child sex and age. Model also included random intercept and random effect for age. Group difference in measured weight is estimated at 18 months. 
TAB LE 2 Characteristics of fully formula feeding children, their mothers and households, from the Infant and Toddler Feeding Practices Study (ITFPS-2) by potential overfeeding status between 1 and 5 months of age ${ }^{a}$

\begin{tabular}{|c|c|c|c|c|}
\hline Variables & $\begin{array}{l}\text { Full sample } \\
N=197079\end{array}$ & $\begin{array}{l}\text { Potential overfeeding } \\
N=73724\end{array}$ & $\begin{array}{l}\text { Non-overfeeding } \\
\mathrm{N}=123355\end{array}$ & $P$-value* \\
\hline \multicolumn{5}{|l|}{ Child, birth factors } \\
\hline Race/ethnicity, n (\%) & & & & .007 \\
\hline Non-Hispanic black & 45079 (22.87) & $14834(20.12)$ & $30245(24.52)$ & \\
\hline Hispanic Spanish speaking & 38001 (19.28) & $8607(11.67)$ & $29394(23.83)$ & \\
\hline Hispanic English speaking & $43410(22.03)$ & $18720(25.39)$ & $24690(20.02)$ & \\
\hline Low birthweight, $\mathrm{n}$ (\%) & $17008(8.63)$ & $3753(5.09)$ & $13255(10.75)$ & .07 \\
\hline$\geq 3$ weeks preterm, $\mathrm{n}(\%)$ & $26456(13.42)$ & $6294(8.54)$ & $20162(16.37)$ & .06 \\
\hline High activity (13 m), n (\%) & 77088 (39.12) & 32505 (44.09) & $44583(36.14)$ & .17 \\
\hline \multicolumn{5}{|l|}{ Maternal, household factors: } \\
\hline Married, n (\%) & $46214(23.45)$ & $15803(21.44)$ & $30411(24.65)$ & .43 \\
\hline Cohabiting with child's father, $\mathrm{n}(\%)$ & 94155 (47.78) & $34006(46.13)$ & $60149(49.23)$ & .50 \\
\hline Overweight or obese, $\mathrm{n}(\%)$ & $109646(55.64)$ & $44046(59.74)$ & $65600(53.18)$ & .20 \\
\hline First pregnancy, n (\%) & 78585 (39.87) & $28331(38.43)$ & $50254(40.74)$ & .65 \\
\hline Any prior breastfeeding, $\mathrm{n}(\%)$ & 80125 (40.66) & $30496(41.37)$ & $49629(40.23)$ & .79 \\
\hline Timing of WIC enrollment, $n$ (\%) & & & & .80 \\
\hline First trimester & $52200(26.49)$ & $20769(28.17)$ & 31431 (25.48) & \\
\hline$<$ High school & $55180(28.00)$ & $16713(22.67)$ & 38467 (31.22) & \\
\hline High school & 73357 (37.22) & $29570(40.11)$ & 43787 (35.54) & \\
\hline >High school & $68386(34.70)$ & 27441 (37.22) & 40945 (33.24) & \\
\hline Receive other benefits, $\mathrm{n}$ (\%) & $169893(86.21)$ & $62959(85.40)$ & $106934(86.69)$ & .73 \\
\hline Household Income, n (\%) & & & & .56 \\
\hline$<75 \% \mathrm{FPL}$ & $134180(68.08)$ & $48033(65.15)$ & $86147(69.84)$ & \\
\hline 75 to $130 \%$ FPL & $45616(23.15)$ & $19130(25.95)$ & $26486(21.47)$ & \\
\hline$>130 \% \mathrm{FPL}$ & $17283(8.77)$ & $6562(8.90)$ & 10721 (8.69) & \\
\hline Household food security, n (\%) & & & & .31 \\
\hline High/marginal & $101501(51.50)$ & $34983(47.45)$ & 66518 (53.92) & \\
\hline Low & $61617(31.27)$ & $24856(33.71)$ & $36761(29.80)$ & \\
\hline Very low & 33961 (17.23) & $13885(18.83)$ & $20076(16.27)$ & \\
\hline
\end{tabular}

Abbreviations: FPL, federal poverty level; WIC, Special Supplemental Nutrition Program for Women, Infants and Children;

${ }^{*} P$-values were determined for the weighted sample by Rao-Scott modified $\chi^{2}$.

${ }^{a}$ Numbers of subjects presented in this table represent the weighted sample incorporating statistical weights to accommodate differential sampling and response rates. 
and standard error (SE) of the mean. Statistical comparisons for characteristics of infants experiencing and not experiencing potential overfeeding in the weighted sample were performed with Rao-Scott modified $\chi^{2}$ tests or F-tests for categorical and continuous variables, respectively. No adjustment was done for multiple testing. ${ }^{28}$ All analyses were performed using SAS 9.3 (SAS Institute, Cary, North Carolina). $P$-values $<.05$ indicate statistical significance.

\section{$3 \mid$ RESULTS}

Of the 197079 fully formula-fed infants in the weighted sample, $73724(37.41 \%, 95 \% \mathrm{Cl}=33.57-41.25 \%)$ were identified as experiencing potential overfeeding between 1 and 5 months of age. Daily kilocalorie consumption was higher at every dietary recall for infants experiencing potential overfeeding than infants not experiencing potential overfeeding from 1 to 36 months of age, with those experiencing potential overfeeding consuming significantly more calories per day ( $26.10 \mathrm{kcal}, P$-value .004$)$ than those not experiencing potential overfeeding in the fully adjusted longitudinal mixed effects model (Figure 1 Panel A, Table 1). Infants experiencing potential overfeeding weighed more than infants not experiencing potential overfeeding at every measurement from 0 to 36 months of age and were significantly heavier $(0.18 \mathrm{~kg}, P$-value .01$)$ in the fully adjusted longitudinal mixed effects model (Figure 1 Panel B, Table 1). Differences for energy intake and weight are reported at 18 months (the mid-point of the age distribution of the measurements).

Few infant characteristics were associated with potential overfeeding (Table 2). Infant race/ethnicity was significantly associated with potential overfeeding with Non-Hispanic White and Englishspeaking Hispanic infants being overrepresented among those experiencing potential overfeeding, representing 38.12 and $25.39 \%$ of infants experiencing potential overfeeding but only 24.55 and $20.02 \%$ of infants not experiencing potential overfeeding, respectively ( $P$ value .007). Potentially overfed infants were more likely than nonoverfed infants to be categorized as high activity at 5 months of age ( 64.49 vs $50.15 \%, P$-value .01), but this activity difference was not observed at 13 months of age. No maternal or household characteristics were associated with potential overfeeding.

No differences were observed between infants experiencing and not experiencing potential overfeeding with regards to bottle feeding practices or the daily frequency of feedings (total and formula) between 1 and 5 months of age (Table 3). No differences were observed for the individuals that the mother spoke to about her feeding decisions or breastfeeding intent (data not shown). Significant associations were identified for mothers' breastfeeding beliefs, with mothers of infants experiencing potential overfeeding less likely than mothers of infants not experiencing potential overfeeding to agree that "when breastfeeding, only the mother can feed the baby" ( 53.24 vs $64.87 \%, P$-value .002$)$ or that "breastfeeding is easier than bottle feeding" (61.99 vs $70.42 \%, P$ value .04). Mothers of infants experiencing potential overfeeding were less likely than mothers of infants not experiencing potential overfeeding to agree that an infant turning away from the bottle indicates the infant
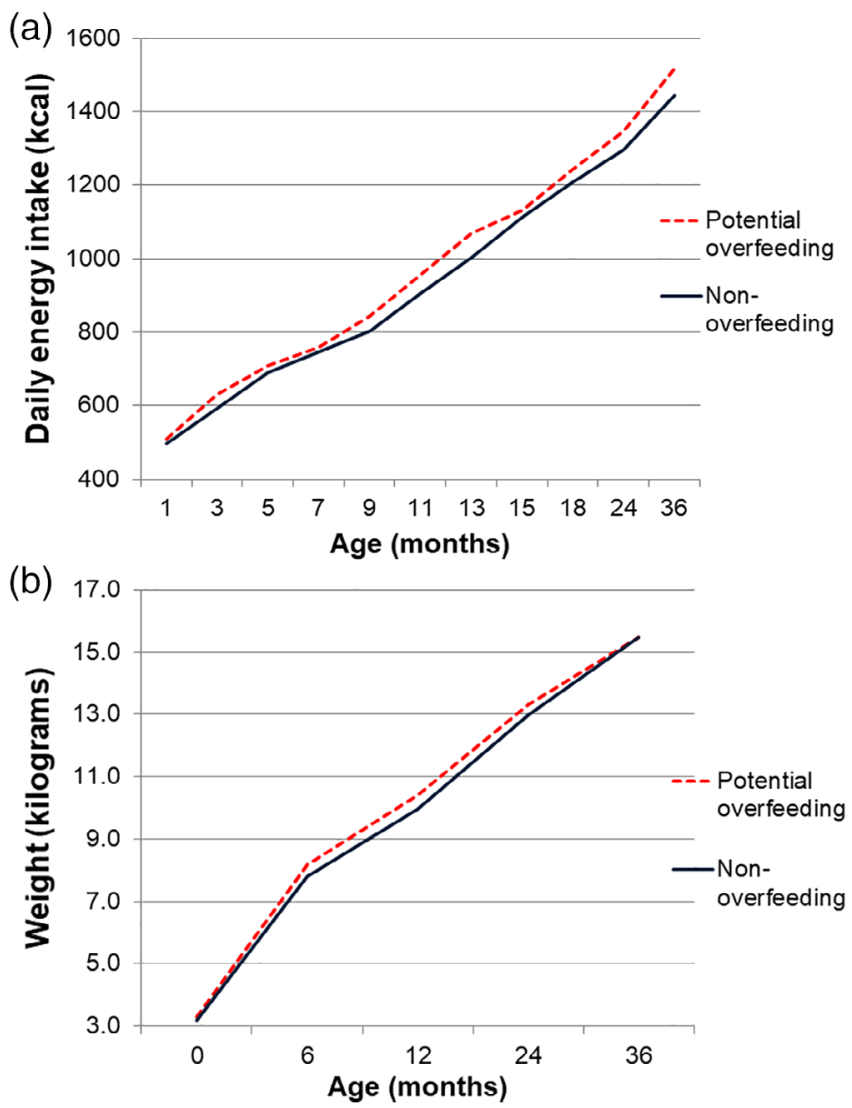

FIGURE 1 Daily energy consumption and weight for women, infants and children (WIC)-participating formula feeding infants experiencing potential overfeeding and non-overfeeding in ITFPS-2. A, Daily caloric intake based upon 24-hour dietary recalls between 1 and 36 months of age. B, Child weight in kilograms between 0 and 36 months of age. Note: Reported daily kilocalories and measured weight in kilograms for 197079 infants in the weighted sample of formula feeding infants, unadjusted for covariates. No statistical test was performed based on these weighted but unadjusted kilocalorie and body-weight values

is full (67.42 vs $76.43 \%, P$-value .04) and that crying always indicates that an infant is hungry (22.05 vs $32.50 \%, P$-value .04$)$. Maternal perception of the amount of formula provided by WIC was significantly associated with potential overfeeding, with mothers of infants experiencing potential overfeeding significantly more likely than mothers of infants not experiencing potential overfeeding to report that WIC provides insufficient formula when their infants were between 1 and 5 months of age (60.31 vs $24.82 \%, P$-value <.0001) or between 7 and 13 months of age (61.58 vs $48.31 \%$, $P$-value .007).

\section{4 | DISCUSSION}

Potential overfeeding during early infancy was identified in $37.41 \%$ of fully formula-fed WIC-participating infants based upon a large longitudinal assessment of infant and toddler feeding practices in the ITFPS2. In longitudinal analyses of repeated dietary recall and weight measurements, infants who experienced potential overfeeding consumed 
TAB LE 3 Maternal reported infant feeding practices and beliefs by potential overfeeding status identified between 1 and 5 months of age ${ }^{a}$

\begin{tabular}{|c|c|c|c|}
\hline Variable & $\begin{array}{l}\text { Potential overfeeding } \\
N=73724\end{array}$ & $\begin{array}{l}\text { Non-overfeeding } \\
\mathrm{N}=123355\end{array}$ & $P$-value* \\
\hline \multicolumn{4}{|l|}{ Feeding practices, $\mathrm{n}(\%)$} \\
\hline Prop bottle up when feeding & $27268(36.99)$ & $37193(30.15)$ & .15 \\
\hline Mix formula with extra water & $6559(8.90)$ & $7805(6.33)$ & .24 \\
\hline Add bottle sweetener & $28159(38.20)$ & $44664(36.21)$ & .71 \\
\hline Extra-large bottle hole & $17918(24.30)$ & $34878(28.27)$ & .35 \\
\hline Feeding on schedule (1-5 m) & $54784(74.31)$ & $88524(71.76)$ & .52 \\
\hline Feeding on schedule (7-13 m) & $45238(61.36)$ & $70960(57.53)$ & .31 \\
\hline Daily formula feedings ( $3 \mathrm{~m}$ ), Mean \pm SEM & $6.64 \pm 0.12$ & $6.64 \pm 0.13$ & 1.00 \\
\hline Daily feedings $(5 \mathrm{~m})$, Mean \pm SEM & $6.79 \pm 0.19$ & $6.85 \pm 0.15$ & .93 \\
\hline Daily formula feedings ( $5 \mathrm{~m}$ ), Mean \pm SEM & $6.22 \pm 0.17$ & $6.30 \pm 0.13$ & .91 \\
\hline \multicolumn{4}{|l|}{ Mom agrees ${ }^{b}$ that..., $\mathrm{n}(\%)$} \\
\hline She plans to use formula & $22775(39.88)$ & $35624(38.60)$ & .91 \\
\hline Breastfeeding is all an infant needs & $43718(78.23)$ & $71098(79.23)$ & .99 \\
\hline If breastfeeding, only mom can feed baby & $29848(53.24)$ & $59867(64.87)$ & .002 \\
\hline She knows baby getting enough with bottle & $50992(90.18)$ & 77643 (84.98) & .38 \\
\hline Breastfeeding is easier & 34097 (61.99) & $63147(70.42)$ & .04 \\
\hline WIC provides insufficient formula (1-5 m), n (\%) & $44465(60.31)$ & $30620(24.82)$ & $<.0001$ \\
\hline WIC provides insufficient formula (7-13 m), n (\%) & $45400(61.58)$ & $59597(48.31)$ & .007 \\
\hline
\end{tabular}

Note: Bold values indicate statistical significance $(P<0.05)$.

Abbreviations: M, months; SEM, SE of the mean; WIC, Special Supplemental Nutrition Program for Women, Infants and Children.

${ }^{*} P$-values were determined for the weighted sample by Rao-Scott modified $\chi^{2}$ or $F$ test for categorical and continuous variables, respectively.

${ }^{a}$ Numbers of subjects presented in this table represent the weighted sample incorporating statistical weights to accommodate differential sampling and response rates.

${ }^{\mathrm{b}}$ Responses to response scales were dichotomized: strongly agree, agree and neutral were combined into one category, while disagree and strongly disagree were combined into a second category.

significantly more calories daily and were significantly heavier than infants who did not experience potential overfeeding. Few infant, maternal, or household characteristics were associated with this potential overfeeding, but Non-Hispanic white and English speaking Hispanic children were more likely to experience potential overfeeding, and infants experiencing potential overfeeding were more likely to be classified as high activity at 5 months of age. Lower percentages of mothers of infants experiencing potential overfeeding agreed that during breastfeeding that they alone could feed their infants, that breastfeeding is the easiest option for feeding their infants, that an infant who turns away from the bottle is full, or that crying always indicates hunger. Mothers of infants experiencing potential overfeeding were significantly more likely to report that WIC provides too little formula in early and late infancy than mothers of infants not experiencing potential overfeeding.

\subsection{Associations between potential overfeeding, energy intake and weight}

Excess energy consumption in formula-fed infants is a problem which contributes to excess weight gain, ${ }^{29}$ and intake was determined to be 
higher than expected energy requirements by an average of $8 \%$ for infants under 6 months of age and by $22 \%$ for infants between 6 and 11 months of age in the Feeding Infants and Toddlers Study 2008. ${ }^{30}$ Fully formula-fed infants experiencing potential overfeeding in this study were found to be consuming 26 additional kilocalories per day compared to fully formula-fed infants not experiencing potential overfeeding. Graulau et al (2019) found that $43.2 \%$ of WIC-participating mother-infant dyads in Hawaii and Puerto Rico who use formula consumed formula above recommendations, ${ }^{31}$ which is similar to the estimate of $37.41 \%$ from this national sample of WIC participants. In contrast to the presently reported association between potential overfeeding and infant weight, the study in Hawaii and Puerto Rico did not identify an association between amount of formula consumed (below recommendation vs at recommendation or above recommendation) and either weight gain during the 4 months follow-up or weight status at the follow-up visit between 4 and 6 months of age. ${ }^{31}$ This difference between the two studies may be due to the small sample size in that study, a follow-up that was too short to detect differences, the categorization of anthropometric outcomes or unmeasured confounding factors. A longitudinal study in the United Kingdom identified associations between the amount of formula (high or low) consumed at 8 months of age and child weight at 18 months of age, ${ }^{32}$ which is similar to the difference in weight identified between infants experiencing and not experiencing potential overfeeding in this study.

\section{2 | Infant and maternal correlates of potential overfeeding}

Promotion of responsive feeding, defined as feeding practices that are infant-led and responsive to infant hunger and satiation cues, has been a primary focus of efforts aimed at reducing overfeeding and rapid weight gain during infancy. ${ }^{33}$ Thus, one potential contributor to excess energy consumption for infants in the present study may have been mothers' misinterpretation of infant cues leading to overfeeding given identified associations between how mothers interpret infant cues and potential overfeeding. Specifically, a smaller proportion of potentially overfed infants had a mother who believed that turning away from a bottle means that the baby is full or that crying always means the baby is hungry. That mothers of potentially overfeeding infants were less likely to interpret crying as a sign of hunger was unexpected, as associations between maternal sensitivity to infant cues during feeding and amount fed to bottle fed infants, ${ }^{34}$ the ability of infants to self-regulate intake, ${ }^{35}$ and healthier infant weight gain ${ }^{36}$ have been reported. However, these findings are consistent with prior research that indicated mothers are less responsive to cues for satiation than hunger. ${ }^{37}$ Taken together, the absence of feeding frequency differences and the lower attribution of crying to hunger suggests that mothers of potentially overfed infants may be adequately distinguishing hunger from other cues, whereas the lesser recognition of turning away from the bottle as a satiation cue suggests potential overfeeding may be attributable to lower responsiveness to infant satiation cues leading to greater intakes during feedings. Alternatively, if mothers terminate feeding in response to infant satiation cues, but do not reduce the amount of formula prepared for feedings, this may contribute to excess discarded formula and misclassification of infants as potentially overfed. Given the limited scope of ITFPS-2 questions on maternal sensitivity and responsiveness to infant hunger and satiation cues, and the correlational nature of this study, further research is needed to better understand the mechanisms underlying these associations.

Another possible explanation for potential overfeeding could be related to errors in formula preparation. Recent research has identified unintentional excess formula dispensed in $78 \%$ of bottles, regardless of size, in a laboratory setting and over-dispensing formula during bottle preparation was more common in caregivers of children and younger adults. ${ }^{38}$ This may suggest that unintentional measurement error while dispensing formula contributes to excess consumption of formula among WIC participants. A study evaluating the readability of instructions on commercial infant formula packaging identified that the directions for preparation and use and storage have an average reading difficulty requiring the equivalent of a college level education. ${ }^{39}$ There was no assessment of maternal literacy in the present study, but there was no association between self-reported maternal educational attainment and potential overfeeding in this study. Because the majority of mothers in this study of a national sample of WIC participants had a high school degree or lower educational attainment, a mismatch between literacy demands of formula packaging and maternal education may contribute to the identified potential overfeeding of infants. Potential overfeeding infants were more likely than non-overfeeding infants to be highly active at 5 months of age, however the association between potential overfeeding and activity dissipated by 13 months of age. It is possible that high activity children demonstrate hunger more often than their less active counterparts, and therefore a proportion of the infants identified as experiencing potential overfeeding may be fed appropriately for their elevated activity level.

The absence of association between potential overfeeding and maternal education and socio-economic status was unexpected. In a prior study among low-income immigrant Latina mothers recruited from WIC centers in the southeastern United States, greater maternal education was significantly associated with healthier feeding practices, ${ }^{40}$ which conflicts with the present findings of no association between potential overfeeding and lower maternal education. This discrepancy could be due to greater detail on the educational attainment of those who did not complete high school in the prior study, or the much smaller proportion of the sample from that study who completed high school or more than high school (19\% and 3\%, respectively) compared to the present study (37\% and $35 \%$ completed high school or more than high school, respectively). Prior research has identified associations between bottle feeding practices such as adding sweetener or cereal to a bottle and overfeeding or weight gain among of formula feeding infants, ${ }^{41}$ however the present study did not identify associations between self-reported bottle feeding practices and whether participating infants experienced potential overfeeding. 


\section{3 $\quad$ Strengths and limitations}

Noteworthy strengths of the present study include the large sample size, the availability of sampling weights ensure the analytic sample is representative of the population of WIC participants arising from WIC agencies large enough to be included in the sampling frame and the high quality of the survey and repeated dietary recalls conducted among participants. Survey questions about maternal perception of the sufficiency of formula provided by WIC and dietary recalls allowed for the validation of identified potential overfeeding, and repeated 24-hour dietary recalls and child weight measurements allowed the evaluation of the longitudinal relationship between potential overfeeding and dietary intake and infant weight. This study also has limitations that deserve mention. This study is observational in design, and many covariates were assessed concurrently with potential overfeeding, making many of the reported associations cross-sectional and precluding strong inferences about underlying mechanisms. Potential overfeeding was defined based upon the infant package received from WIC at 1 month of age and whether the mother reported getting formula from WIC and another source when her infant was 1, 3 or 5 months of age. While the infant package received by WIC-participants has been identified as a valid proxy for formula intake, ${ }^{42}$ it is not a direct measurement of diet. Additionally, it is possible that a mother reports getting formula from WIC and another source not because she is feeding the infant more than is recommended, but because some formula provided by WIC was wasted during preparation of bottles. The amount of formula obtained from sources other than WIC was not assessed. Important dyad characteristics, such as infant cue clarity and temperament, were not assessed in this study. Information on physical restriction of the infant was not available, and data on infant activity were limited. Future research should attempt to address these limitations.

\section{5 | CONCLUSIONS}

Over $37 \%$ of WIC-participating infants receiving the fully formulafeeding infant package may be consuming too much formula during the first 6 months after birth. A majority of mothers of infants experiencing potential overfeeding believe WIC provides insufficient formula in both early and late infancy. However, the fully formulafeeding infant package provided by WIC is designed to provide enough formula to meet all known nutritional needs for the infants receiving it between 0 and 5.9 months of age. Findings suggest an opportunity for more education of WIC-participating mothers on the nutritional needs of their formula-fed infants. These educational opportunities can be informed by previous attempts to encourage healthier bottle feeding practices among WIC participants. ${ }^{43}$ More detailed education for formula feeding mothers about responding to satiation cues and how to minimize formula wasted during bottle preparation may reduce the number of women reporting that WIC provides too little formula.

\section{ACKNOWLEDGEMENTS}

S.E.W. conceptualized the study, with input from C.E.A. and A.K.V. C. E.A. and C.E.M. carried out the data management and statistical analysis. All authors were involved in the interpretation of the results. C.E. A. drafted the manuscript, and all other authors edited the manuscript. All authors approved the final version for submission and agree to be responsible for its contents.

\section{CONFLICT OF INTEREST}

C.E.A., C.E.M. and A.K.V. have no conflicts of interest to declare.

S.E.W. reported grant funding from USDA FNS during the study period.

\section{ORCID}

Christopher E. Anderson (D) https://orcid.org/0000-0003-0912-6884

\section{REFERENCES}

1. Polk S, Thornton RJ, Caulfield L, Munoz A. Rapid infant weight gain and early childhood obesity in low-income Latinos and non-Latinos. Public Health Nutr. 2016;19(10):1777-1784.

2. Caprio S. Insulin resistance in childhood obesity. J Pediatr Endocrinol Metab. 2002;15(Suppl 1):487-492.

3. Muntner P, He J, Cutler JA, Wildman RP, Whelton PK. Trends in blood pressure among children and adolescents. JAMA. 2004;291(17): 2107-2113.

4. Singh AS, Mulder C, Twisk JW, van Mechelen W, Chinapaw MJ. Tracking of childhood overweight into adulthood: a systematic review of the literature. Obes Rev. 2008;9(5):474-488.

5. Ogden CL, Carroll MD, Lawman HG, et al. Trends in obesity prevalence among children and adolescents in the United States, 19881994 through 2013-2014. JAMA. 2016;315(21):2292-2299.

6. Pan L, Freedman DS, Sharma AJ, et al. Trends in obesity among participants aged 2-4 years in the special supplemental nutrition program for women, infants, and children-United States, 2000-2014. MMWR Morb Mortal Wkly Rep. 2016;65(45):1256-1260.

7. Lobstein T, Jackson-Leach R, Moodie ML, et al. Child and adolescent obesity: part of a bigger picture. Lancet. 2015;385(9986):2510-2520.

8. Horta BL, Loret de Mola C, Victora CG. Long-term consequences of breastfeeding on cholesterol, obesity, systolic blood pressure and type 2 diabetes: a systematic review and meta-analysis. Acta Paediatr. 2015;104(467):30-37.

9. Azad MB, Vehling L, Chan D, et al. Infant feeding and weight gain: separating breast Milk from breastfeeding and formula from food. Pediatrics. 2018;142(4):e20181092.

10. Papoutsou S, Savva SC, Hunsberger M, et al. Timing of solid food introduction and association with later childhood overweight and obesity: the IDEFICS study. Matern Child Nutr. 2018;14(1):e12471.

11. Oliveira V, Frazao E. The WIC Program: Background, Trends and Economic Issues, 2015 Edition, EIB-134. Washington, DC: US Department of Agriculture, Economic Research Service; 2015

12. Wilde $P$, Wolf A, Fernandes M, Collins A. Food-package assignments and breastfeeding initiation before and after a change in the special supplemental nutrition program for women, infants, and children. Am J Clin Nutr. 2012;96(3):560-566.

13. Chaparro MP, Wang MC, Anderson CE, Crespi CM, Whaley SE. The association between the 2009 WIC food package change and early childhood obesity risk varies by type of infant package received. $J$ Acad Nutr Diet. 2020;120(3):371-385.

14. Taylor J. Updating the WIC food packages: it's about time. Issue Brief George Wash Univ Natl Health Policy Forum. 2006;816:1-14. 
15. Almeida R, Alvarez Gutierrez S, Whaley SE, Ventura AK. A qualitative study of breastfeeding and formula-feeding mothers' perceptions of and experiences in WIC. J Nutr Educ Behav. 2020;S1499-4046(19):31142-X.

16. Harrison GG, Hirschman JD, Owens TA, McNutt SW, Sallack LE. WIC infant and toddler feeding practices study: protocol design and implementation. Am J Clin Nutr. 2014;99(3):742s-746s.

17. May L, Borger C, Weinfield N, et al. WIC Infant and Toddler Feeding Practices Study-2: Infant Year Report. Alexandria, VA: Food and Nutrition Service, USDA; 2017.

18. Birrell CL, Steel DG, Batterham MJ, Arya A. How to use replicate weights in health survey analysis using the National Nutrition and Physical Activity Survey as an example. Public Health Nutr. 2019;22 (18):3315-3326.

19. Saylor J, Friedmann E, Lee HJ. Navigating complex sample analysis using national survey data. Nurs Res. 2012;61(3):231-237.

20. Institute of Medicine of the National Academies, Food and Nutrition Board, 2005. WIC food packages: Time for a change. https://www. fns.usda.gov/wic/wic-food-packages-time-change. Published 2005 Accessed March 11, 2020.

21. Moshfegh AJ, Rhodes DG, Baer DJ, et al. The US Department of Agriculture Automated Multiple-Pass Method reduces bias in the collection of energy intakes. Am J Clin Nutr. 2008;88(2):324-332.

22. Au LE, Gurzo K, Paolicelli C, Whaley SE, Weinfield NS, Ritchie LD. Diet quality of US infants and toddlers 7-24 months old in the WIC infant and toddler feeding practices Study-2. J Nutr. 2018;148(11): 1786-1793.

23. Whaley SE, Whaley M, Au LE, Gurzo K, Ritchie LD. Breastfeeding is associated with higher retention in WIC after age 1. J Nutr Educ Behav. 2017;49(10):810-816. e811.

24. Nommsen-Rivers LA, Cohen RJ, Chantry CJ, Dewey KG. The Infant Feeding Intentions scale demonstrates construct validity and comparability in quantifying maternal breastfeeding intentions across multiple ethnic groups. Matern Child Nutr. 2010;6(3):220-227.

25. Huybrechts I, De Bacquer D, Cox B, et al. Variation in energy and nutrient intakes among pre-school children: implications for study design. Eur J Public Health. 2008;18(5):509-516.

26. Chaparro MP, Crespi CM, Anderson CE, Wang MC, Whaley SE. The 2009 Special Supplemental Nutrition Program for Women, Infants, and Children (WIC) food package change and children's growth trajectories and obesity in Los Angeles County. Am J Clin Nutr. 2019; 109(5):1414-1421.

27. VanderWeele TJ. Principles of confounder selection. Eur J Epidemiol. 2019;34(3):211-219.

28. Rothman KJ. No adjustments are needed for multiple comparisons Epidemiology. 1990;1(1):43-46.

29. Romera G, Figueras J, Rodriguez-Miguelez JM, Ortega J, Jimenez R. Energy intake, metabolic balance and growth in preterm infants fed formulas with different nonprotein energy supplements. J Pediatr Gastroenterol Nutr. 2004;38(4):407-413.

30. Butte NF, Fox MK, Briefel RR, et al. Nutrient intakes of US infants, toddlers, and preschoolers meet or exceed dietary reference intakes. J Am Diet Assoc. 2010;110(12) Suppl:S27-S37.

31. Graulau RE, Banna J, Campos M, Gibby CLK, Palacios C. Amount, preparation and type of formula consumed and its association with weight gain in infants participating in the WIC program in Hawaii and Puerto Rico. Nutrients. 2019;11(3):695.

32. Hopkins D, Steer CD, Northstone K, Emmett PM. Effects on childhood body habitus of feeding large volumes of cow or formula milk compared with breastfeeding in the latter part of infancy. Am J Clin Nutr. 2015;102(5):1096-1103.

33. Perez-Escamilla R, Segura-Perez S, Lott M, et al. Feeding Guidelines for Infants and Young Toddlers: A Responsive Parenting Approach. http://healthyeatingresearch.org. Published 2017. Accessed March 30, 2020.

34. Ventura AK, Hernandez A. Effects of opaque, weighted bottles on maternal sensitivity and infant intake. Matern Child Nutr. 2019;15(2): e12737.

35. DiSantis KI, Hodges EA, Johnson SL, Fisher JO. The role of responsive feeding in overweight during infancy and toddlerhood: a systematic review. Int J Obes (Lond). 2011;35(4):480-492.

36. Farrow $\mathrm{C}$, Blissett J. Does maternal control during feeding moderate early infant weight gain? Pediatrics. 2006;118(2):e293-e298.

37. Hodges EA, Johnson SL, Hughes SO, Hopkinson JM, Butte NF, Fisher JO. Development of the responsiveness to child feeding cues scale. Appetite. 2013;65:210-219.

38. Altazan AD, Gilmore LA, Guo J, et al. Unintentional error in formula preparation and its simulated impact on infant weight and adiposity. Pediatr Obes. 2019;14(12):e12564.

39. Wallace LS, Rosenstein PF, Gal N. Readability and content characteristics of powdered infant formula instructions in the United States. Matern Child Health J. 2016;20(4):889-894.

40. Cartagena D, Ameringer SW, McGrath JM, Masho SW, Jallo N Myers BJ. Factors contributing to infant overfeeding in lowincome immigrant Latina mothers. Appl Nurs Res. 2015;28(4): 316-321.

41. Appleton J, Russell CG, Laws R, Fowler C, Campbell K, DenneyWilson E. Infant formula feeding practices associated with rapid weight gain: a systematic review. Matern Child Nutr. 2018;14(3): e12602.

42. Whaley SE, Koleilat M, Jiang L. WIC infant food package issuance data are a valid measure of infant feeding practices. J Hum Lact. 2012;28(2):134-138.

43. Kavanagh KF, Cohen RJ, Heinig MJ, Dewey KG. Educational intervention to modify bottle-feeding behaviors among formula-feeding mothers in the WIC program: impact on infant formula intake and weight gain. J Nutr Educ Behav. 2008;40(4):244-250. 